International Journal of Computer Science \& Information Technology (IJCSIT) Vol 3, No 5, Oct 2011

\title{
An IR-based Pedestrian Detection System Implemented with Matlab-Equipped Laptop and Low-Cost Microcontroller
}

\author{
Erwin Thomas Gilmore, Chukwunweike Ugbome, and Charles Kim \\ Department of Electrical and Computer Engineering \\ Howard University \\ Washington, DC \\ luckyprince50@yahoo.com; ethomasg@gmail.com; ckim@howard.edu
}

\begin{abstract}
Infrared (IR)-based human detection research has received much attention within the research community in recent years. Recently, for industrial applications, infrared (IR) sensors have been applied to human detection in vehicle safety, night-vision, and military applications. They directly detect targets with warm temperatures in the image, providing a potentially simpler and quicker solution to human detection, especially during nighttime. This paper report a project of developing a pedestrian detection system in urban environments implemented with a Matlab on a laptop platform interfaced with a low-end microcontroller. This system primarily focuses on digital, infrared imagery and pedestrian candidate selection and detection using available detection algorithms, and on simple, economical implementation which is possible through the direct interfacing between the algorithm in Matlab and low-end external hardware for indication of the detection result.
\end{abstract}

Keywords- IR-Camera, Embedded Computing, Pedestrian Detection, Infrared Imagery, Human Cadidiate Selection, Microcontrooler

\section{INTRODUCTION}

Infrared (IR) sensors have been applied to human detection in some applications such as vehicle safety, night-vision, and military applications. They directly detect targets with warm temperatures in an image, providing a potentially simpler and quicker solution to human detection, especially during nighttime, despite their higher cost and less affordable than optical cameras with comparable resolutions. IR-based human detection has been investigated by a number of groups. Their research focus is on human detection in an urban environment on the street or on campus to provide assistance to the drivers or for surveillance purposes, especially during the evening hours [1 - 7]. Compared to non-urban environment where terrain, mountain, and/or forest scenes are the main background, urban scenes usually have artificial objects in their background such as buildings and streetlights whose temperatures are generally elevated during the evening. Vehicles also generate heat that can show up as hotspots in IR images. These background noises can make IR-based human detection more complicated. On the other hand, however, pedestrians on the street are generally in simple walking or standing upright poses which are easier to recognize than other complicated poses such as stretching (e.g., running and bending) or hiding, which can often occur in a non-urban environment such as in the battlefield. It is obviously a more challenging situation and new methods have to be introduced in dealing with the unrestricted human poses. Since little research has been done for human detection in such a non-urban 
International Journal of Computer Science \& Information Technology (IJCSIT) Vol 3, No 5, Oct 2011

environment, previously, we analyzed many existing algorithms designed for pedestrian detection in urban environment, and experimentally evaluated them against non-urban IR images [8]. It was shown that, as expected, these existing algorithms performed especially poorly on humans in stretching or hiding poses because they relied heavily on features of standing or walking human shapes and appearances. As a result, humans with stretching poses or partial occlusions (such as behind the trees) in the IR images were mostly missed.

In this paper, we present a development project of a pedestrian detection system for unban environment implemented with a Matlab equipped laptop interfaced with a low-cost microcontroller and trivial indicators. This system primarily focuses on digital, infrared imagery and its use for pedestrian detection utilizing available detection. Additionally, it focuses on an economical implementation of the system via simple connection of the imagery and the algorithms with a low-end low-cost microcontroller. The target performance is to achieve a near real-time pedestrian detection and monitoring capability and to demonstrate live at the conclusion of the project. The simple interfacing to external microcontroller from a Matlab equipped laptop is another motivation for this project since the previous researches of the authors in image processing and feature detection have been mostly remained mostly inside the laptop in simulation mode [9]. The possibility of connecting the developed algorithms to external hardware devices provides excitement and high expectation.

The final system of the project is intended to give a twenty four hour and seven day per week real time monitoring of activities on our pedestrians and subsequently to provide information for planning. In this project, the presence of a human operator is not required for making decisions or viewing the infrared images on a monitor. Instead, a simple indicator is connected the microcontroller to display the decision made by the Matlab implemented algorithm. The indicator can be replaced by a means of communication, wired and/or wireless, for to give information for security watch or decisions.

This paper is organized as follows. Section II summarily reviews the related works. Section III briefs IR technology and IR-based pedestrian feature selection. Next, the architectural description of the system design and implementation is discussed in Section IV. Then, Section $\mathrm{V}$ describes, the test and evaluation of the developed system. Lastly, Section VI concludes the project description of the paper.

\section{RELATED WORKS}

Pedestrian detection, human imagery or object tracking have received wide research attention in the past years and are still serious area of research activities today [10, 11]. Various authors have been able to accurately and effectively present different ways to detect and track the pedestrian systems [12 - 17]. Here we summarize pedestrian detection and tracking approaches at diverse locations using different methodologies. A vision-based approach, adopted a dynamical graph matching approach to identify pedestrians efficiently and automatically [11]. In another, the extraction of the contour of a target was utilized by a coding method for pedestrian detection [10]. In parallel, pedestrian detection and tracking algorithms have found many important applications from surveillance to planning and intelligent systems [18 - 21]. In particular, a generalized EM algorithm was adopted for pedestrian detection and tracking in infrared imagery using shape for dynamic background mosaic [23]. On the other hand, a multistage infrared stationary human detection has also been examined for various urban and indoor 
International Journal of Computer Science \& Information Technology (IJCSIT) Vol 3, No 5, Oct 2011

settings using FLIR imagery [24]. This multi-stage detection algorithm consists of a set of intuitive image features and two distinct feed-forward neural networks that are cascaded together. The efficient feature extractors in the first stage quickly process the whole input scene and identify potential target areas for further scrutiny.

Although these research areas have gained significant success over the years, they do not have wide application and external communication capabilities compared to our work as tests shows that our work has very wide range of application and attractive flexibility. In addition, the reported algorithms do not have wide automatic decision making capabilities. This paper presents a cost effective and broader ways of implementing a Matlab algorithm of an infrared based pedestrian detection. This algorithm is coded such that the detected objects are analyzed automatically and decisions are made based on the automatic analysis. The detected objects are classified as correct detection, correct rejection and false detection. The uniqueness of the proposed approach is such that these classified detections are simply communicated via simple external interfaces depending on areas of interest, for security watch, officers can be dispatched based on the detected interests.

\section{IR-BASED FEATURE SELECTON AND DETECTION}

The use of cameras in surveillance is increasing in the last years due to the low cost of the sensor and the requirement by surveillance in public places. Based on the distribution of infrared radiation spectrum, an IR sensor can be classified as one of the following four categories according to its wavelength: Short Wave IR (SWIR) of $0.7-3 \mu \mathrm{m}$; Mid Wave IR (MWIR) of $3-6 \mu \mathrm{m}$; Long Wave IR (LWIR) of $6-15 \mu \mathrm{m}$; and Far IR (FIR) of $15-1000$ $\mu \mathrm{m}$. IR energy is emitted from all material and objects above $0^{\circ} \mathrm{K}$ as thermal radiation. The upper limit of FIR occurs in a region where it is difficult to envision the output from a source as heat (peak radiation occurs at $3^{\circ} \mathrm{K}$ ). At normal temperature, human body radiates most strongly at about $10 \mu \mathrm{m}$, which apparently corresponds to the wavelength range of LWIR. As a result, LWIR, MWIR and some FIR sensors are usually used for human detection in most applications.

In digital imagery processing, the process of human feature selection consists mainly of three steps. First, preprocessing is done to obtain hotspots from the image using the methods of histogram equalization and segmentation by a threshold approach. A threshold approach is a technique often used to separate foreground targets from background environment based on their differences in image intensity. In a simple threshold approach, a single intensity threshold is used to generate a binary image from the original image. For example, the intensity threshold can be determined using the following equation:

$$
\text { Threshold }=\alpha I_{\text {mean }}+\beta I_{\max },
$$

where $\alpha$ and $\beta$ satisfy $\alpha+\beta=1$, and they represent weights assigned to the mean intensity $I_{\text {mean }}$ and the maximum intensity $I_{\max }$ of the original image. Determination of appropriate values for the weights, however, is not a trivial task. It is usually dependent on the specific setting of the IR camera such as brightness and/or contrast. For our project, from the IR test images, we selected the weight for best performance as: $\alpha=0.4$ and $\beta=0.6$.

Second, morphological operations are conducted to suppress background noises. To remove isolated noises in the threshold binary images, we used morphological operations of combined erosion and dilation with a $2 \times 2$ erosion followed by a $5 \times 4$ dilation and, after these, most of the 
International Journal of Computer Science \& Information Technology (IJCSIT) Vol 3, No 5, Oct 2011

scattered background noises disappeared in the resulting image. This not only suppressed background noise but also aggregated the regions of interest (ROI) which represent to candidate pedestrians and non-pedestrian objects.

Third, selection of human feature is the last step in determining a feature using metrics such as aspect ratio constraint, local histogram filtering, and/or morphological human model matching. In our project, determination of whether a hotspot was pedestrian or not was made by computing their bounding boxes (height and width) and eliminating nonhuman candidates by size and special aspect ratio (ratio of height and width) of the bounding boxes. The aspect ratio of 0.25 was experimentally selected to allow more versatility by accommodating pedestrians in various standing poses. All the steps described above are implemented in the Matlab environment in a laptop.

\section{IR-BASED PEDESTRIAN DETECTION SYSTEM}

The architecture of the developed system is as follows. The Matlab-equipped Laptop is the processor of feature selection and detection of the IR-based pedestrian detection system. Digital images are supplied by an infrared camera interfaced to the laptop's peripheral component interface (PCI) card. Formatting and displaying the images are also handled by the laptop. Final decision of pedestrian or non-pedestrian is also performed by the Matlab in the laptop. In other words, all the necessary steps and processes are conducted and implemented the Matlabequipped laptop platform. The low-end, low-cost microcontroller, Basic Stamp 2 ("BS2") is to serially connect to the laptop for indicating the result made by the laptop platform. BS2 is a stamp-sized 8-bit microcontroller which includes a peripheral interface controller chip, flash memory and EEPROM for code storage, RAM for data handling, and numerous input/output interface terminals. The convenient programming environment of BS2 is made possible by preprogrammed BASIC interpreter in the flash memory with built-in functions which allows users to write a code easily without a steep learning curve. The serial communication (RS232) function of the Matlab and the convenient coding environment of BS2 make the interface simple and economical. Also, with ample amount of interface terminals, BS2 provides an easy way to connect an indicator (such as LEDs of different color, green for pedestrian and red for nonpedestrian, for example). Figure 1 depicts the schematic of the pedestrian detection system.

The laptop platform used is an HP Compaq TC4400 Tablet PC with WindowsXP operating system, and the IR camera used is a ThermoVision A320 InfraRed camera from FLIR Systems. The image capture card is the ImperX Image Capture Card model VCE-B5A01. The Matlab is the 7th version (R14) wit service pack 3 installed. The BS2 used in the project is Board of Education (USB) with Basic Stamp II Module from Parallax. The indicator consists of 2 LEDs, one green and the other red, each connected through a $470 \mathrm{ohm}$ resistor from an output pin of the BS2.

Numerous test images were produced for initial analysis for intensity weight determination and familiarization of the types of image generated and processed from the system. Figures 2(a) and 2(b) are just two of such test images. Each image was generated at a rate of 1 image frame a second. Figure 2(a) shows a person standing with hands down while Figure 2(b) depicts a person with hands raised above the shoulders. A picture frame number is indicted at the upper right corner of each figure, and a spot temperature in Celsius at the top left corner. 


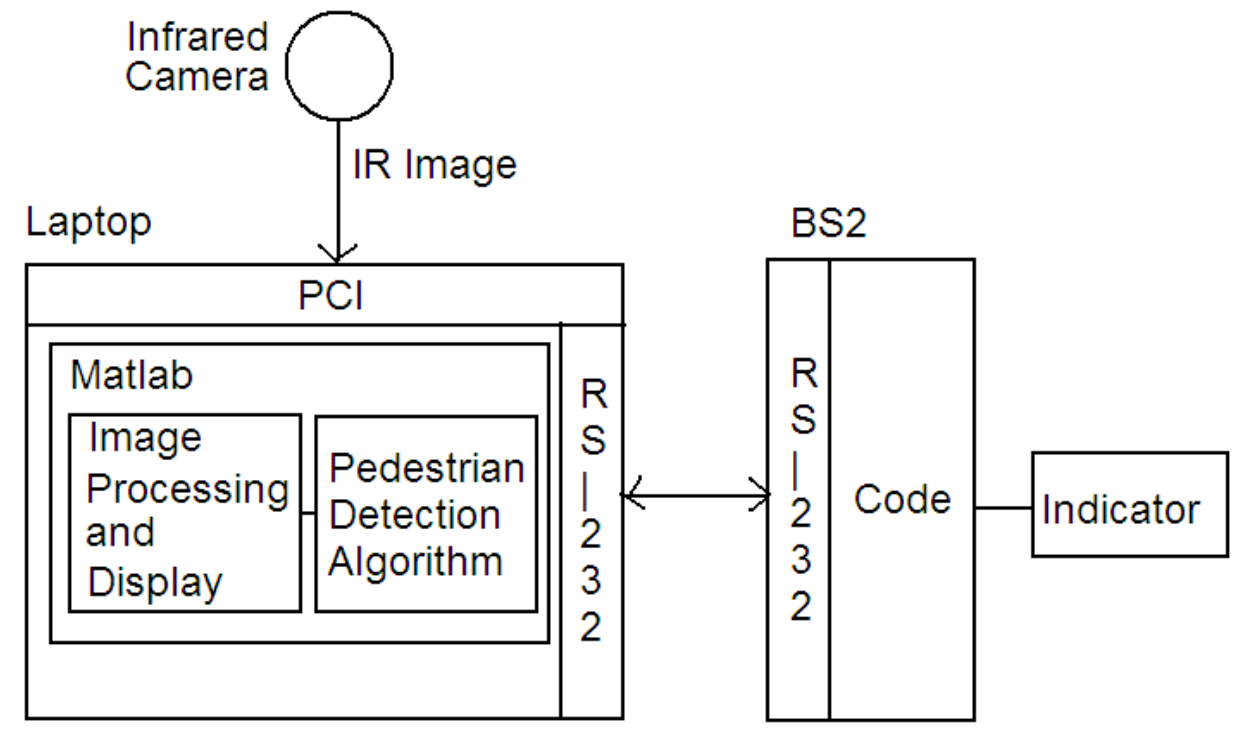

Figure1. Schematic of the Pedestrian Detection System

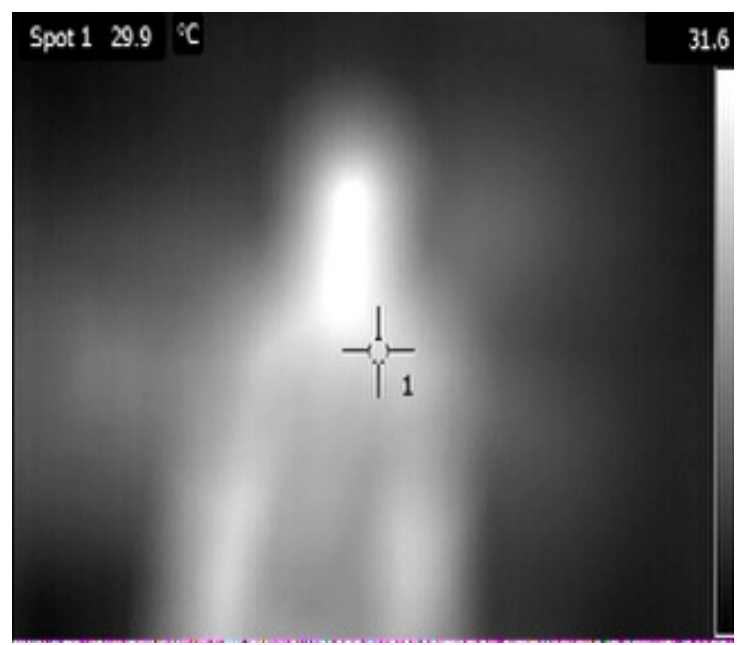

(a)

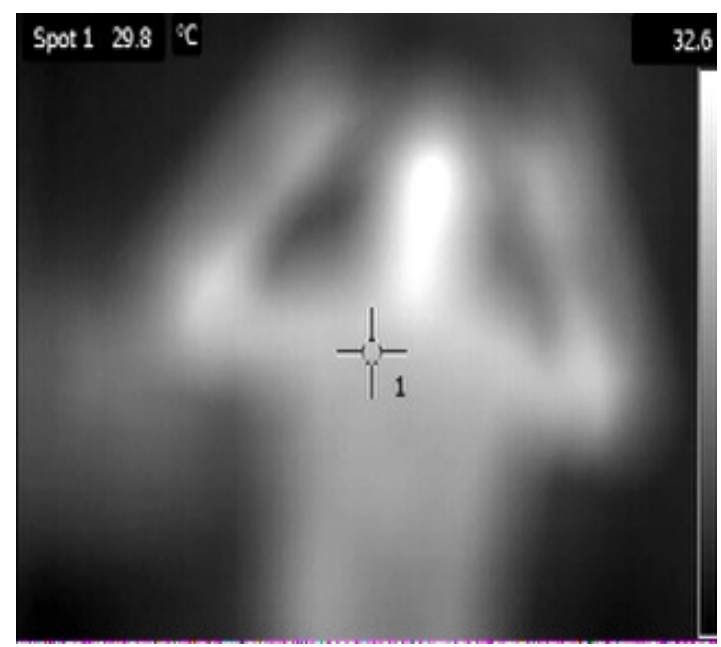

(b)

Figure 2. Two test images, (a) and (b).

\section{TESTING AND EVALUATION OF THE DETECTION SYSTEM}

This section discusses the testing and overall performance of our system. The tests were conducted in two different ways: off-line and on-line tests. The off-line test was done on the detection algorithm using a set of test images to benchmark the performance and the on-line test to demonstrate the functionality of the entire system in real-time operation. The evaluation of the tests was made to assess the strengths and weaknesses of the pedestrian detection system. 
The test images for the off-line test were generated using the same IR camera used to conduct the on-line real-time test. Approximately 520 IR images were produced each with a resolution of 320x240 pixels. Figures 3(a) and 3(b) show examples of off-line test. Figure 3(a) shows the case of successful pedestrian detection and rejection of non-human among the identified regions of interest. The Correct Detection is denoted by the green bounding box in the middle and the Correct Rejection is highlighted in red bounding boxes at the upper left corner. For each correct detection, a detection signal was sent via MATLAB to the BS2, and BS2 in turn on the green LED. On the other hand, Figure 3(b) demonstrates a case with a False Alarm. As can be see, there is an incorrect classification of a detected human body part object (an arm) seen in the middle of the image as well as a Correct Detection for the same person at the left.

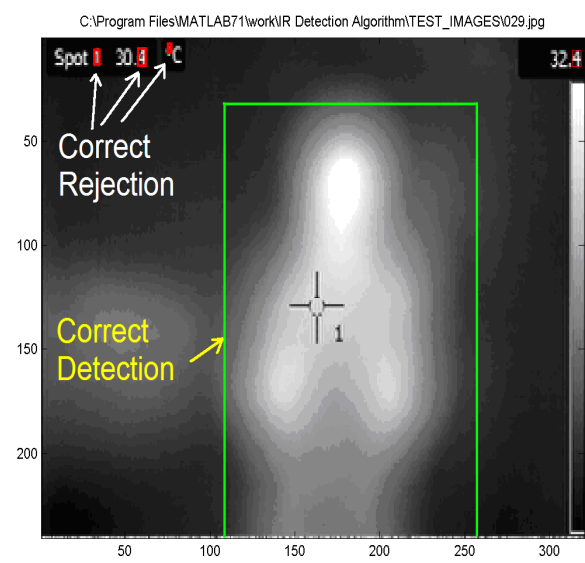

(a)

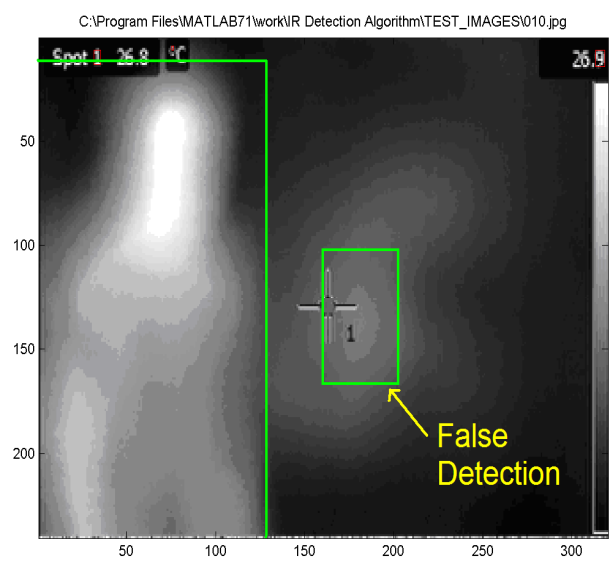

(b)

Figure 3. Off-line test examples depicting (a) correction detection and correct rejection and (b) false detection.

The online performance test of the algorithm was done with the IR camera providing the realtime images while the Matlab code handled the online image processing and formatting, with the rate of one image per second. The online test revealed that the algorithm has strength in not only detecting images but the ability to detect and differentiate human from non-humans. The detection result was similarly provided from the laptop to the BS2 so that BS2 indicated the result. Figure 4 shows a picture of the system set-up under online testing. The camera was facing toward the area of the open space and provided images of object (not seen in the picture) in the space. The image processed in a second was displayed one at a time. 
International Journal of Computer Science \& Information Technology (IJCSIT) Vol 3, No 5, Oct 2011

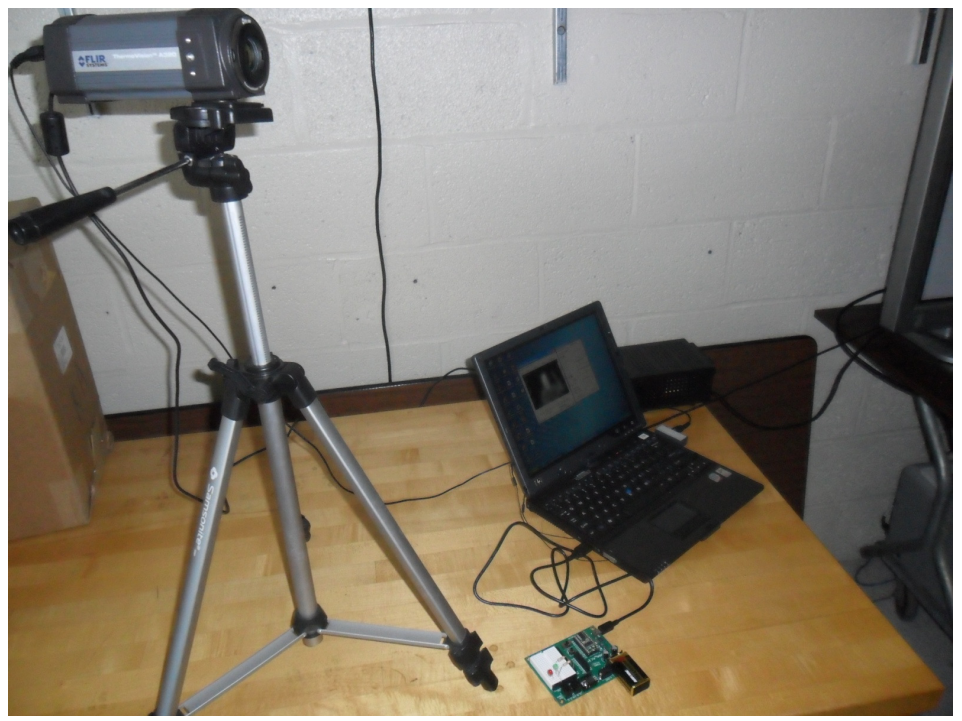

Figure 6. A picture of system set-up under online testing.

From the on-line testing, a receiver operating characteristics (ROC) curves were produced to evaluate the performance of the algorithm and the entire detection system. For the ROC curves, we first generated two separate data sets by taking images at different times of a person in slightly different positions. Each data set had 90 images from which 550 regions of interests were determined. The resulting 2 curves of ROC are represented in Figure 6. Each curve shows that the correct detection rate is approximately $90 \%$ at a false alarm rate of $10 \%$.

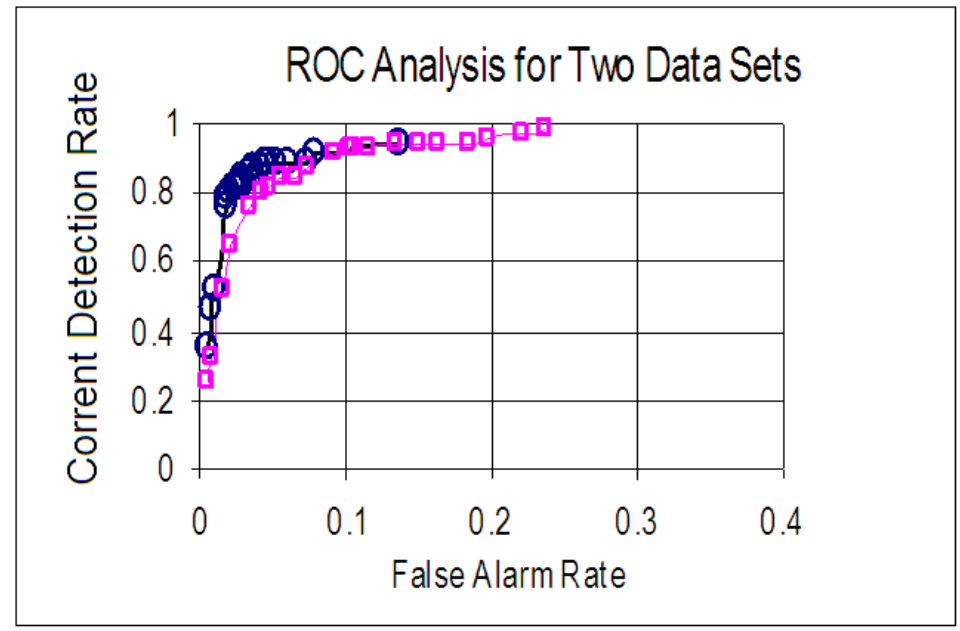

Figure 7. ROC curves for two data sets.

From the performance test, our approach of solution implementation has a few strengths. First, the use of a simple platform with Matlab and low-microcontroller is successful in doing all necessary steps of on-line and off-line data processing, formatting, image detection and 
International Journal of Computer Science \& Information Technology (IJCSIT) Vol 3, No 5, Oct 2011

differentiation. Second, the implementation is unique with the combination of its design simplicity, flexibility, and efficiency in operations. However, our pedestrian detection has a few weak points, too. The detection algorithm may not perform as well for more complex imagery, i.e. two or more people within an image. Because the detection algorithm is dependent on aspect ratios of the individual detected hot spots, further work may need to be done to accommodate more complex human poses, e.g. lying down, squatting, kneeling, etc. For improvement, we think that the detection performance can be improved by using a more advanced classification scheme such as a Support Vector Machine (SVM) or even a neural network. In addition, the speed of image processing could be much improved if a new tool of image processing such as cellular automata could be adopted for low level image processing and feature detection. Both of the above are currently being studied.

\section{CONCLUSIONS}

A development project of Infrared (IR)-based pedestrian detection system implementation was described. The detection was developed using the available detection algorithms on a laptop platform with Laptop, and its detection result was interfaced to external, low-end microcontroller via serial communication link. The detection performance of the developed system in ROC analysis showed, in both off-line and on-line images, an acceptable rate of $90 \%$ correct detection at a false classification rate of $10 \%$. Future studies will focus on using a Cellular Automata based image processing and detection scheme to improve performance in detection accuracy and computational speed.

\section{REFERENCES}

[1] M. Bertozzi, A. Broggi, A. Fascioli, T. Graf, and M.M. Meinecke, "Pedestrian detection for driver assistance using multiresolution infrared vision", IEEE Trans. on Vehicular Technology, 53 (6), 2004.

[2] F. Xu, X. Liu, and K. Fujimura, "Pedestrian detection and tracking with night vision", IEEE Trans. on Intelligent Transportation Systems, 6 (1), 2005.

[3] M. Yasuno, N. Yasuda, and M. Aoki, "Pedestrian detection and tracking in far infrared images", Proc. 2004 IEEE Computer Society Conference on Computer Vision and Pattern Recognition Workshops (CVPRW"04), 2004.

[4] Y. Fang, K. Yamada, Y. Ninomiya, B. K. P. Horn, and I. Masaki, "A shape-independent method for pedestrian detection with far-infrared images", IEEE Trans. on Vehicular Technology, 53 (6), 2004.

[5] X. Liu and K. Fujimura, "Pedestrian detection using stereo night vision", IEEE Trans. on Vehicular Technology, 53 (6), 2004.

[6] C. Dai, Y. Zheng, and X. Li, "Layered representation for pedestrian detection and tracking in infrared imagery", Proc. IEEE Computer Society Conference on Computer Vision and Pattern Recognition, Volume 3, pp. 20-26, 2005.

[7] M. Bertozzi, A. Broggi, C. Hilario Gomez, R.I. Fedriga, G. Vezzoni, and M. Del Rose, "Pedestrian detection in far infrared images based on the use of probabilistic templates", Proc. 2007 IEEE Intelligent Vehicles Symposium, 2007.

[8] J. Zeng, A. Sayedelah, M. Chouikha, T. Gilmore, and P. Frazier, "Infrared Detection of Humans in Stretching Poses Using Heat Flow,” ICASSP 2008. 
International Journal of Computer Science \& Information Technology (IJCSIT) Vol 3, No 5, Oct 2011

[9] E. Thomas Gilmore, Preston D. Frazier, and M. F. Chouikha, "Improved Human Detection Using Image Fusion," Proceedings of the IEEE ICRA 2009 Workshop on People Detection and Tracking, Kobe, Japan, May 2009.

[10] J. HeikkilGa, O. SilvHen, A real-time system for monitoring of cyclists and pedestrians, IEEE Proceedings on Visual Surveillance, Fort Collins, Colorado, 1999, pp. 74-81.

[11] C.J. Pai, H.R. Tyan, Y.M. Liang, H.Y. Mark Liao, S.W. Chen, Pedestrian detection and tracking at crossroads, Proceedings of the International Conference on Image Processing, Barcelona, Spain, September 14-17, 2003.

[12] I. Kakadiaris, D. Metaxas, Model-based estimation of 3D human motion, IEEE Trans. Pattern Anal. Mach Intell. 22 (2000) 1453-1459.

[13] J. Deutscher, A. Blake, I. Reid, O. Oxford, Articulated body motion capture by annealed particle filtering, in: Proc. IEEE Conf. on Computer Vision and Pattern Recognition, 2000.

[14] Y. Song, X. Feng, P. Perona, Toward detection of human motion, in: Proc. IEEE conference on Computer Vision and Pattern Recognition, 2000.

[15] A. Mohan, T. Poggio, Example-based object detection in images by components, IEEE Trans. Pattern Anal. Mach. Intell. 23 (2001) 349-361.

[16] R. Cutler, L. Davis, Robust real-time periodic motion detection analysis and applications, IEEE Trans. Pattern Anal. Mach. Intell. 22 (2000) 781-797.

[17] U. Franke, D. Gavrila, S. Go“ rzig, F. Linder, F. Paetzold, C. Wo“ hler, Autonomous driving goes downtown, IEEE Intell. Syst. Appl. 13 (1998) 32-40.

[18] B. Leibe, et al., Pedestrian detection in crowded scenes, in: Proc. Of IEEE Conf. Computer Vision and Pattern Recognition, 2005.

[19] P. Viola, M. Jones, D. Snow, Detecting pedestrians using patterns of motion and appearance, in: Proc. IEEE Conf. Computer Vision,2003.

[20] I. Kakadiaris and D. Metaxas, Model-based estimation of 3D human motion, IEEE Trans. Pattern Anal. Mach Intell. 22 (2000), pp. 1453-1459.

[21] J. Deutscher, A. Blake, I. Reid, O. Oxford, Articulated body motion capture by annealed particle filtering, in: Proc. IEEE Conf. on Computer Vision and Pattern Recognition, 2000.

[22] J. Zeng, A. Sayedelahl, M. F. Chouikha, E. T. Gilmore, and P. D. Frazier, "Human Detection in Non-Urban Environment Using Infrared Images," Proc. Int. Conf. Information, Communications, and Signal Processing, pp.1-4, Singapore, Dec 2007.

[23] Congxia Dai, Yunfei Zheng and Xin Li "Pedestrian detection and tracking $\mathrm{n}$ infrared imagery using shape and appearance" 106 (2007) 288-299.

[24] Alex Lipchem Chan, US. Army research laboratory, Adelphi "Multi-stage infrared stationary human detection"04. 